\title{
Patient needs and medication styles in
} COPD

\author{
L.M. Osman* and M.E. Hyland ${ }^{\#}$
}

\begin{abstract}
Patient self-care in illnesses such as chronic obstructive pulmonary disease (COPD) is influenced by "trade-offs" that patients make between their social and psychological needs, and the "best practice" clinical management of their illness. Patients weigh the benefits of taking medication against the costs, such as symptoms.

Personal styles of medication use and decision-making also affect how well COPD patients respond to treatment plans and their acceptance of particular treatments, including inhalers. The large amount of information now available may have made patients more autonomous than before, but the actual locus of control varies widely between patients. Patients seem to take a pragmatic approach when assessing inhaler devices, basing preference on how effective they perceive the device to be. Patients with COPD show loyalty to devices and, in the main, try to comply with instructions given by the healthcare provider.

The health path for chronic obstructive pulmonary disease, which describes the course the disease typically takes, is a progressive deterioration. Patients therefore adopt a conservative attitude to their health goals and what they expect to gain from treatment and management plans. An understanding of these influences will help healthcare providers support the patient better and give the appropriate advice at each phase of a patient's health path.
\end{abstract}

KEYWORDS: Attitudes, chronic obstructive pulmonary disease, inhaler, medication, patient his paper focuses on patient selfmanagement in respiratory illness within a framework of patient decision-making. Important aspects that influence decision-making are the "trade-offs" patients are willing to make to satisfy social and psychological needs (which may appear to be at odds with clinical "best practice ${ }^{\prime \prime}$ ), personal styles of self-care, and the health path of chronic obstructive pulmonary disease (COPD) as a chronic illness.

\section{TRADE-OFF IN ILLNESS MANAGEMENT}

In everyday decision-making, we commonly trade off the advantages and disadvantages when reaching a final decision about a necessary choice. Patients apply such trade-offs when deciding how they will manage both short-term and chronic illnesses. They will see any treatment available for a condition as having psychological costs as well as therapeutic benefits. The concept of patients trading-off costs and benefits when managing their disease is frequently employed in "willingness to pay" studies, which measure preference for one attribute of a treatment over another, or readiness to avoid comorbidities of a given disease [1].

The benefits afforded by treatment are more than symptom relief alone. They also include the extent to which the treatment helps patients regain what they regard as "normality", and the degree to which it reduces their fears of what will happen in the future path of their illness. For patients with COPD, preventing variation in their illness by controlling the risk of exacerbation is also a benefit, which may not be directly related to the efficacy of the treatment in relieving everyday symptoms. Patients may accept or trade off a lower level of functioning, or even the risk of side-effects, if they are confident that they will prevent major exacerbations. The value that each patient places on the outcome of a particular treatment intervention is very personal and can be difficult for health professionals and caregivers to predict. In a study of elderly patients with chronic illnesses that included COPD, the level of agreement between patient and caregiver about the acceptability of a particular health outcome was poor [2].

Psychological costs of treatment include the patient's dislike of regular medication use [3]. In respiratory medicine, this aversion is separate from, and appears to be more powerful than, that felt towards inhaled corticosteroids [3, 4]. Patients are also reluctant to be "dependent" on medication, and fear the long-term effects of
AFFILIATIONS

*Chest Clinic, Aberdeen Royal

Infirmary, Aberdeen, and

\#University of Plymouth, Plymouth, UK.

CORRESPONDENCE

L.M. Osman

Senior Research Fellow

Chest Clinic

Aberdeen Royal Infirmary

Foresterhill

Aberdeen

AB25 2ZN

UK

Fax: 441224551210

E-mail: med078@abdn.ac.uk 
taking any medication [5]. They may perceive medication use as carrying a stigma because of the need for use in public (e.g. inhalers), and may then fail to take the treatment as agreed. When trading-off leads to patients using treatment in ways that are not clinically standard, patients are often described as non-compliant, although in practice these non-standard treatment regimens may have been explicitly or implicitly agreed between health professionals and their patients.

A study of the use of oxygen therapy by COPD patients [6] illustrates the types of trade-off that patients feel this treatment requires from them. The study described these patients as struggling to balance their need for optimal treatment benefits against the perceived social costs of oxygen therapy. Many patients felt that increased use of oxygen therapy required significant compromises, notably the sacrifice of some important quality-of-life aspects. Perceived costs included feelings of self-consciousness and stigmatisation, fear of side-effects, dependency and increased functional limitation.

Another example of trade-off can be seen in the difficulty respiratory patients have in giving up smoking. Smoking cessation practice acknowledges that this is not just due to the difficulty of breaking a physiological addiction; patients perceive real benefits of smoking that will be lost if they stop [7]. The social and psychological benefits of smoking that they perceive outweigh both the long-term health gains (which, even though they are acknowledged by the patient to be rational, may seem too distant or abstract) and, in the case of COPD, the even more abstract concept that the long-term deterioration in lung function will be slowed down, but not reversed, by stopping smoking. The ratio of positive to negative aspects of smoking predicts readiness to quit [8]. Similarly, the positive and negative aspects of medication perceived by patients can predict adherence to prescribed medication, to a greater extent than clinical and sociodemographical factors [9].

An example of trading-off relating to inhaler therapy, albeit in patients with asthma rather than COPD, comes from a recently published small study carried out in Sheffield, UK, which looked at how asthma patients used chamber devices with metered-dose inhalers [10]. The competency with which 63 outpatients used a range of inhalers was assessed. As expected, competence was poor for standard pressurised metered-dose inhalers (pMDIs) but good for breath-actuated pMDIs and dry powder inhalers. The addition of a spacer device to a pMDI resulted in $100 \%$ of the patients being rated as competent. However, although these patients were all fully competent in the use of a pMDI plus spacer (and thus able to gain real clinical benefit from it), the overwhelming majority $(80 \%)$ did not use their chamber device. Patients traded-off clinical effectiveness for ease of use.

Among asthma patients, we have found that some symptoms are weighted more negatively than others, and that when asked to choose a preferred "symptom week", patients would accept (trade-off) increased sleep disturbance in return for reduced cough [11]. JOHANSSON et al. [12] found that although patients preferred combined inhalers, they would be willing to trade-off this preference if they could gain increased symptomfree days.

\section{PATIENT STYLES IN SELF-CARE}

Over the past two decades, qualitative studies have shown that patients have idiosyncratic and personal styles of self-care. An awareness of these possible styles can help generate understanding of the rationale behind what may seem undesirable self-care patterns. In the 1980s, CONRAD [13] investigated styles of medication management in people using anti-epilepsy medication. The author identified several management styles, including "testing", "pragmatic use", "confident and autonomous", "denial" and "chaotic". The self-care style of the patient affects the "locus of control" between the patient and clinician, i.e. the extent to which a patient accepts that the doctor is in charge of health decisions. The increasing availability of large amounts of medical information online means that many patients are now better informed about recent medical developments and feel more confident about taking a greater role in decisions about their management and treatment. The use of regular medication by testers fluctuates because they continually assess whether they still need medication by deliberately stopping taking it. The pragmatic users also take their medication irregularly because they develop a personal management plan that they believe is effective for them and which does not necessitate using medication continuously. They believe that they know when they can safely reduce or stop using preventive medication. When considered in relation to the previous discussion regarding trade-off, it seems reasonable to suspect that the testers and pragmatic users are likely to be more tolerant of risk than patients who continue to use medication even when symptom-free.

In contrast to such a confident or autonomous style, another management style that has been identified in many studies of patient self-care is denial [14]. In a study of primary care patients, Dowell and HUDSON [15] suggested that patients' acceptance of a treatment is closely linked to how fully they accept the illness. Finally, a chaotic personal style has been identified in the way that some patients manage their respiratory illness [16]. Personal styles can fluctuate over time, and can also be related to the stage of the illness or treatment. It has been suggested that many patients go through a testing phase in the early stages after a treatment has been prescribed or changed.

\section{PATIENT ATTITUDES TO INHALER DEVICES}

COPD patient focus groups [17] have shown that patients evaluate the effectiveness of inhalers and can judge whether or not their medication is effective. Many patients taking inhaled bronchodilators perceive that their efficacy is variable; these fluctuations are probably representative of day-to-day variations in the pathophysiology of the patient's disease in response to environmental exposures. This presents a problem to patients, however, as it causes them to doubt the effectiveness of the inhaler. The perceived effectiveness of their medication is important to patients; feedback from focus groups conducted in the USA with patients of all ages suggests that patients who were offered a more aesthetically pleasing device than their own would not pay a premium for it unless it would definitely improve the standard of their treatment (personal communication: M. Hyland). Patients thus seem to treat their inhalers pragmatically; their purpose is to deliver medication rather than just to feel or look good. 
Patients develop loyalty to a particular device and, due to familiarity, prefer that device to others, even if they may not be fully competent in using it. For example, some patients actually like the feeling of the aerosol from a pMDI hitting the back of their throat (the "cold Freon" effect) and believe this means that the drug is "going down", even though drug depositing on the throat does not reach the lungs. Research from focus groups suggests that intentional non-compliance in COPD patients is low [17]. Indeed, preliminary results of a recently completed postal survey of over 300 COPD patients being treated in UK general practice [18] suggest that the majority of COPD patients tried to take their inhalers or medicines exactly as instructed by their clinician or nurse. Nevertheless, high rates of compliance or adherence to inhaled medication regimens will not produce better outcomes if devices are not used competently.

\section{HEALTH PATHS}

The goals of each patient are shaped and limited by how they perceive the prognosis for their condition. The likely course of the disease (or "health path") may therefore influence the patient's responses and the style of illness management. For instance, the typical health path of moderate asthma (fig. 1a) is from fluctuation in the early stages when management is being established, to stability with good functional capacity when management has been resolved [19]. The COPD path, however, differs from the moderate asthma path because it is an inherently deteriorating trajectory (fig. 1b), however well
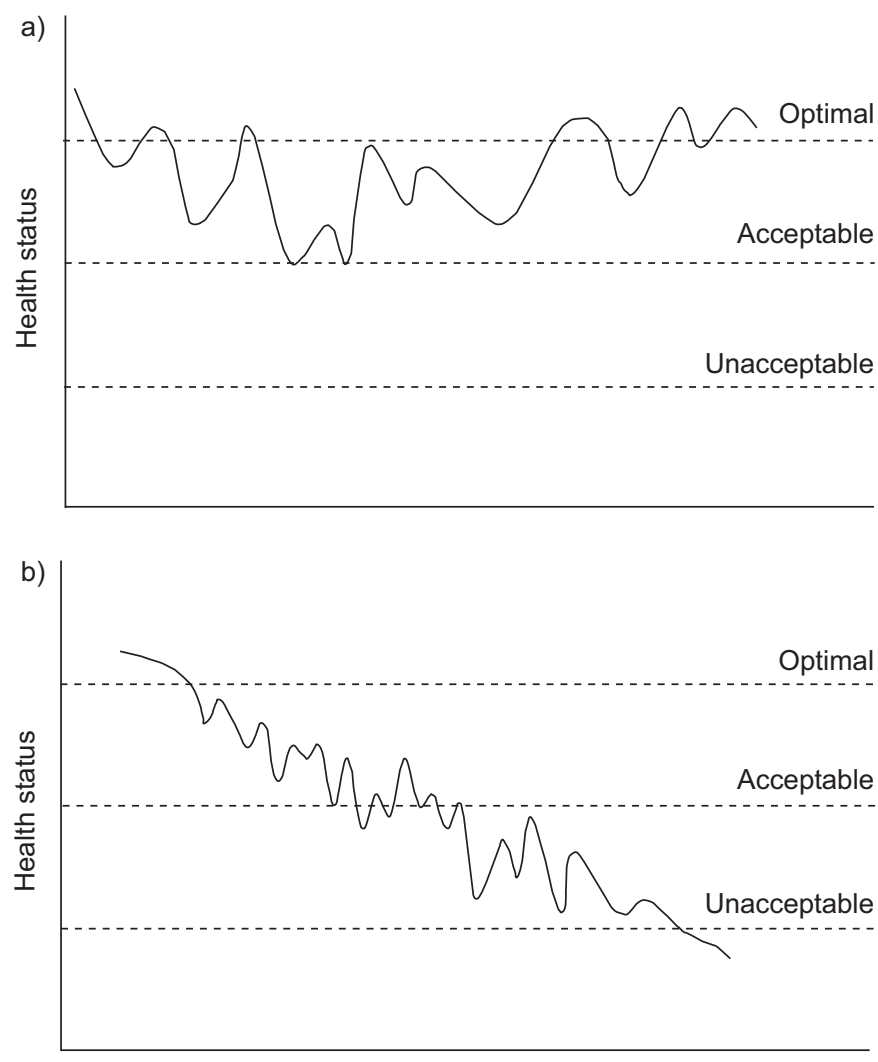

Time

FIGURE 1. Typical health paths for a) asthma and b) chronic obstructive pulmonary disease managed the disease [19]. The constant deterioration in the COPD health path means that the concept of returning to "normality" (as a perceived benefit of treatment) is not really achievable in the same way as for asthma patients.

Whereas the goals in asthma are to achieve a level as near to full functioning as possible, the goals in COPD are to palliate symptoms and slow down deterioration [20]. Regardless of treatment, patients experience inexorable loss in COPD. Whilst many people with asthma can successfully achieve full functioning and do not feel limited by their disease, typical positive responses in COPD patients are acceptance, stoicism and determination. Qualitative studies have found that individuals try to hold on to quality of life through strategies that compensate for the limitations imposed on them by their illness [19].

These characteristic paths and goals of the different illnesses will influence the decision-making behaviour and styles of management of patients. It is likely that chronic obstructive pulmonary disease patients will be more conservative in their approach to treatment and may have more anxieties about it. However, the typical stoicism of chronic obstructive pulmonary disease patients may make them tolerant of less-thanoptimal treatment.

\section{SUMMARY}

- The need of chronic obstructive pulmonary disease patients for independence, control of their condition and maintenance of social functioning may conflict with their need to control their symptoms. Occasionally, some patients will trade-off symptom control in order to satisfy these psychological needs.

- Patients with chronic obstructive pulmonary disease react to their disease in different ways and adopt a variety of self-care styles in how they manage it. Recognising these personal styles will assist in effective management.

- The typical health path for a chronic obstructive pulmonary disease patient is a long-term deterioration with little or no chance of returning to "normality". This often makes patients conservative and stoical about their health goals and their expectations of treatment benefits.

- Patients with chronic obstructive pulmonary disease seem to take a pragmatic approach towards choice of inhaler devices and usually base their preference on how effective the drug-device combination is perceived to be.

\section{REFERENCES}

1 French MT, Mauskopf JA. A quality-of-life method for estimating the value of avoided morbidity. Am J Public Health 1992; 82: 1553-1555.

2 Fried TR, Bradley EH, Towle VR. Valuing the outcomes of treatment: do patients and their caregivers agree? Arch Intern Med 2003; 163: 2073-2078.

3 Osman LM, Russell IT, Friend JA, Legge JS, Douglas JG. Predicting patient attitudes to asthma medication. Thorax 1993; 48: 827-830.

4 van Grunsven PM, van Schayck CP, van Kollenburg HJ, et al. The role of "fear of corticosteroids" in nonparticipation 
in early intervention with inhaled corticosteroids in asthma and COPD in general practice. Eur Respir J 1998; 11: 1178-1181.

5 Hansson SM, Lowhagen O. Drug compliance and identity: reasons for non-compliance. Experiences of medication from persons with asthma/allergy. Patient Educ Couns 2004; 54: 3-9.

6 Earnest MA. Explaining adherence to supplemental oxygen therapy: the patient's perspective. J Gen Intern Med 2002; 17: 749-755.

7 Lennox AS, Osman LM, Reiter E, et al. Cost effectiveness of computer tailored and non-tailored smoking cessation letters in general practice: randomised controlled trial. BMJ 2001; 322: 1396.

8 Etter JF, Humair JP, Bergman MM, Perneger TV. Development and validation of the Attitudes Towards Smoking Scale (ATS-18). Addiction 2000; 95: 613-625.

9 Horne R, Weinman J. Patients' beliefs about prescribed medicines and their role in adherence to treatment in chronic physical illness. J Psychosom Res 1999; 47: 555-567.

10 Brennan VK, Osman LM, Graham H, Critchlow A, Everard ML. True device compliance: the need to consider both competence and contrivance. Respir Med 2005; 99: 97-102.

11 Osman LM, McKenzie L, Cairns J, et al. Patient weighting of importance of asthma symptoms. Thorax 2001; 56: 138-142.
12 Johansson G, Stallberg B, Tornling G, et al. Asthma treatment preference study: a conjoint analysis of preferred drug treatments. Chest 2004; 125: 916-923.

13 Conrad P. The meaning of medications: another look at compliance. Soc Sci Med 1985; 20: 29-37.

14 Adams S, Pill R, Jones A. Medication, chronic illness and identity: the perspective of people with asthma. Soc Sci Med 1997; 45: 189-201.

15 Dowell J, Hudson H. A qualitative study of medicationtaking behaviour in primary care. Fam Pract 1997; 14: 369-375.

16 Harris GS, Shearer AG. Beliefs that support the behavior of people with asthma: a qualitative investigation. J Asthma 2001; 38: 427-434.

17 Jones RCM, Hyland ME, Hanney K, Erwin J. A qualitative study of compliance with medication and lifestyle modification in chronic obstructive pulmonary disease (COPD). Primary Care Respiratory Journal 2004; 13: 149-154.

18 Jones RCM, Hyland ME, Hanney KE. Preliminary data from the Lung Information Needs Questionnaire (LINQ). Primary Care Respiratory Journal 2005; 14: 270-271.

19 Cicutto L, Brooks D, Henderson K. Self-care issues from the perspective of individuals with chronic obstructive pulmonary disease. Patient Educ Couns 2004; 55: 168-176.

20 Seamark DA, Blake SD, Seamark CJ, Halpin DM. Living with severe chronic obstructive pulmonary disease (COPD): perceptions of patients and their carers. An interpretative phenomenological analysis. Palliat Med 2004; 18: 619-625. 